\title{
ON THE HYPERBOLICITY AND THE SCHOTTKY PROPERTY OF COMPLEX SPACES
}

\author{
DO DUC THAI
}

(Communicated by Clifford J. Earle, Jr.)

\begin{abstract}
The purpose of this paper is to study the hyperbolicity and the tautness of spaces that have the Schottky property. Moreover, the hyperbolicity of compact complex spaces is characterized by the classical theorem of Bloch.
\end{abstract}

\section{INTRODUCTION}

In [4] Hahn and Kim considered complex manifolds satisfying the Schottky property and claimed that a complex manifold $M$ is hyperbolic if and only if $M$ has the Schottky property (see [4, Theorem 1]). However, that theorem is not true.

In addition, the authors stated that a compact complex manifold $M$ is hyperbolic if and only if every map $f$ in $\operatorname{Hol}(D, M)$ is Bloch (see [4, Theorem 2]). However, their proof of this theorem uses a lemma for which they gave an incorrect proof (see $[4, \S 3])$.

The aims of this paper are to present counterexamples to Theorem 1 in [4] and to give a correct proof of Theorem 2 in [4].

The author is grateful to Professors N. V. Khue and M. G. Zaidenberg for their help and to the referee for many valuable comments.

\section{COMPleX SPACES SATISFYING THE SCHOTTKY PROPERTY}

We first give the following

1.1. Definition. Let $M$ be a complex space and $h_{M}$ a hermitian metric on the tangent space $T M$ of $M$. Assume that $\rho_{M}$ is the distance function (or the integrated metric) associated with $h_{M}$. The space $M$ is said to have the Schottky property for $h_{M}$ if for each $p \in M$, each relatively compact open set $W$ in a coordinate neighbourhood of $p$, and each $r \in(0,1)$, there exists a positive constant $S=S(W, r)$ such that every map $f \in \operatorname{Hol}(D, M)$ with $f(0) \in W$ satisfies $\rho_{M}(p, f(z)) \leq S$ for $|z| \leq r$, where $D$ is the open unit disc in $\mathbb{C}$ and $\operatorname{Hol}(D, M)$ denotes the space of holomorphic maps from $D$ into $M$ equipped with the compact-open topology.

We now present counterexamples to Theorem 1 in [4].

Received by the editors October 10, 1990 and, in revised form, March 31, 1992.

1991 Mathematics Subject Classification. Primary 32H20, 32H25; Secondary 32H15. 
1.2. Counterexamples. (1) By the continuity of distance functions, every compact complex space $M$ satisfies the Schottky property for any hermitian metric $h_{M}$ on $T M$. Taking $M$ to be complex projective space, we see that having the Schottky property does not imply that $M$ is hyperbolic.

(ii) Let $M$ be a bounded domain in $\mathbb{C}^{n}$ that is not a domain of holomorphy, and let $h_{M}$ be a complete hermitian metric on $T M$.

Then $M$ is hyperbolic, but $M$ does not satisfy the Schottky property for $h_{M}$.

Indeed, under the assumption that $M$ satisfies the Schottky property, we will prove that $\operatorname{Hol}(D, M)$ is a normal family. This will mean that $M$ is taut [1, Theorem 2, p. 430] and hence, a domain of holomorphy [10, Theorem 2, pp. 211-212], which is a contradiction.

Let $\left\{f_{n}\right\}$ be a sequence in $\operatorname{Hol}(D, M)$ that is not compactly divergent. By definition this means that there are compact subsets $K$ of $D$ and $K^{\prime}$ of $M$ such that $f_{n}(K) \cap K^{\prime} \neq \varnothing$ for infinitely many $n$. Thus there are a subsequence $\left\{f_{n_{k}}\right\}$ and points $z_{k} \in K$ such that $f_{n_{k}}\left(z_{k}\right) \in K^{\prime}$ for all $k$. We can assume that the sequence $\left\{z_{k}\right\}$ converges to a point $z_{0} \in K$ and the sequence $\left\{f_{n_{k}}\left(z_{k}\right)\right\}$ converges to a point $p \in K$. For $k=0,1,2, \ldots$ set

$$
\beta_{k}(z)=\frac{z+z_{k}}{1+\bar{z}_{k} z} \text {. }
$$

Then [8, p. 13] $\beta_{k}$ is a holomorphic automorphism of $D$ and $\beta_{k}$ converges to $\beta_{0}$ as $k \rightarrow \infty$. Put $g_{k}=f_{n_{k}} \circ \beta_{k}$ for all $k \geq 1$. Since the sequence $\left\{g_{k}(0)\right\}=$ $\left\{f_{n_{k}}\left(z_{k}\right)\right\}$ converges to $p$, we can assume that there is a relatively compact open set $W$ in a coordinate neighbourhood of $p$ such that $g_{k}(0) \in W$ for all $k \geq 1$. For each fixed $z \in D$, the Schottky property implies that the sequence $\left\{g_{k}(z)\right\}$ is contained in the compact ball $\left\{x \in M: \rho_{M}(p, x) \leq S(W,|z|)\right\}$. Now $\left\{g_{k}\right\}$ is obviously equicontinuous with respect to the Kobayashi distance $d_{M}$, which defines the underlying topology on $M$ (see [2] or [8, Theorem I.2.3, pp. 19-20]). Hence by the Ascoli-Arzela theorem we may assume that $\left\{g_{k}\right\}$ converges to a map $g$ in $\operatorname{Hol}(D, M)$. Then the subsequence $\left\{f_{n_{k}}\right\}$ converges to the map $g \circ \beta_{0}^{-1}$ in $\operatorname{Hol}(D, M)$. Thus $\operatorname{Hol}(D, M)$ is a normal family, as claimed.

1.3. Remark. The domain $M$ in counterexample (ii) has the Schottky property for the hermitian metric induced by the canonical metric of $\mathbb{C}^{n}$. This shows that the Schottky property of a complex space can depend on the choice of the hermitian metric.

By an analogous proof as in Counterexample 1.2(ii), we have the following

1.4. Proposition. Let $M$ be a hyperbolic complex space and $h_{M}$ a complete hermitian metric on TM. Then $M$ is taut if and only if $M$ has the Schottky property for $h_{M}$.

\section{THE HYPERBOLICITY OF COMPACT COMPLEX SPACES}

First we recall the following

2.1. Definition (see [4]). Let $M$ be a complex space and $h_{M}$ a hermitian metric on $T M$. A map $f \in \operatorname{Hol}(D, M)$ is said to be Bloch if

$$
\sup \left\{\left(1-|z|^{2}\right) \cdot h_{M}\left(f(z), f^{\prime}(z)\right): \quad z \in D\right\}<\infty .
$$


We now prove the following (see [4, Theorem 2])

2.2. Theorem. A compact complex space $M$ is hyperbolic if and only if every map $f \in \operatorname{Hol}(D, M)$ is Bloch.

Proof. $(\Rightarrow)$ Assume that $M$ is hyperbolic. By [7, Theorem 8.1, p. 378], $\operatorname{Hol}(D, M)$ is compact; thus $R=\sup \left\{h_{M}\left(g(0), g^{\prime}(0)\right): g \in \operatorname{Hol}(D, M)\right\}<\infty$. Let $f \in \operatorname{Hol}(D, M)$ and $z \in D$. Set

$$
\beta(w)=\frac{w+z}{1+\bar{z} w} .
$$

Then $[8$, p. 13] $\beta$ is a holomorphic automorphism of $D$, so $g=f \circ \beta \in$ $\operatorname{Hol}(D, M)$. The chain rule gives

$$
R \geq h_{M}\left(g(0), g^{\prime}(0)\right)=\left(1-|z|^{2}\right) \cdot h_{M}\left(f(z), f^{\prime}(z)\right),
$$

which shows that $f$ is Bloch.

$(\Leftarrow)$ Let $f: \mathbb{C} \rightarrow M$ be a holomorphic map. Assume that $p, q \in \mathbb{C}$. Consider two sequences $\left\{z_{n}\right\},\left\{z_{n}^{\prime}\right\}$ of $D$ such that $z_{m} \neq z_{n}^{\prime}$ for all $m, n \geq 1$, $\lim _{n \rightarrow \infty} z_{n}=\lim _{n \rightarrow \infty} z_{n}^{\prime}=1$, and $\lim _{n \rightarrow \infty} d_{D}\left(z_{n}, z_{n}^{\prime}\right)=0$. By a theorem of Weierstrass, there exists a holomorphic map $\gamma: D \rightarrow \mathbb{C}$ such that $\gamma\left(z_{n}\right)=p$, $\gamma\left(z_{n}^{\prime}\right)=q$ for all $n \geq 1$. Put $g=f \circ \gamma \in \operatorname{Hol}(D, M)$. Since $g$ is Bloch, there exists a positive constant $c_{g}>0$ such that

$$
h_{M}\left(g(z), g^{\prime}(z)\right) \leq c_{g} /\left(1-|z|^{2}\right) \text { for all } z \in D .
$$

By integrating both sides of $(*)$ along the geodesic curve between two $z_{n}$ and $z_{n}^{\prime}$ in $D$ and using the definition of the integrated distance $\rho_{M}$, we have $\rho_{M}\left(g\left(z_{n}\right), g\left(z_{n}^{\prime}\right)\right) \leq c_{g} \cdot d_{D}\left(z_{n}, z_{n}^{\prime}\right)$ for all $n \geq 1$. Hence $\rho_{M}(f(p), f(q))=0$ or $f(p)=f(q)$. Thus $M$ contains no nontrivial complex lines. By a theorem of Brody (see [3] or [8, Theorem 2.1, p. 68]), $M$ is hyperbolic.

\section{REFERENCES}

1. T. Barth, Taut and tight complex manifolds, Proc. Amer. Math. Soc. 24 (1970), 429-431.

2. (1972), 439-441.

3. R. Brody, Compact manifolds and hyperbolicity, Trans. Amer. Math. Soc. 235 (1978), 213-219.

4. T. K. Hahn and T. K. Kim, Hyperbolicity of a complex manifold and other equivalent properties, Proc. Amer. Math. Soc. 91 (1984), 49-53.

5. P. Kiernan, On the relations between taut, tight and hyperbolic manifolds, Bull. Amer. Math. Soc. 76 (1970), 49-51.

6. S. Kobayashi, Hyperbolic manifolds and holomorphic mappings, Dekker, New York, 1970.

7. _ Intrinsic distances, measures and geometric function theory, Bull. Amer. Math. Soc 82 (1976), 357-416.

8. S. Lang, Introduction to complex hyperbolic spaces, Springer-Verlag, New York, 1987.

9. H. L. Royden, Remarks on the Kobayashi metric, Lecture Notes in Math., vol. 185, Springer-Verlag, New York, 1971, pp. 125-137.

10. H. Wu, Normal families of holomorphic maps, Acta Math. 119 (1967), 193-233.

Department of Mathematics, Pedagogical Institute of Hanoi, Vietnam 